Sharif University of Technology
Scientia Iranica
SCIENTIA

\title{
Improved CT image reconstruction through partial Fourier sampling
}

\author{
H. Abbasi, Z. Kavehvash* and M. Shabany \\ Department of Electrical Engineering, Sharif University of Technology, Iran, Tehran.
}

Received 7 November 2015; received in revised form 11 January 2016; accepted 21 August 2016

\section{KEYWORDS}

Computerized

tomography;

Compressive sensing;

Partial Fourier;

Systematic sampling.

\begin{abstract}
A novel CT imaging structure based on Compressive Sensing (CS) is proposed. The main goal is to mitigate the $\mathrm{CT}$ imaging time and, thus, $\mathrm{X}$-ray radiation dosage without compromising the image quality. The utilized compressive sensing approach is based on radial Fourier sampling. Thanks to the intrinsic relation between captured radon samples in a CT imaging process and the radial Fourier samples, partial Fourier sampling could be implemented systematically. This systematic compressive sampling helps in better control of required conditions such as incoherence and sparsity to guarantee adequate image quality in comparison to previous CS-based CT imaging structures. Simulation results prove the superior quality of the proposed approach (about $4 \%$ improvement in peak signal-to-noise ratio), achieving the smallest $\mathrm{CT}$ scan time and the best image quality.

(C) 2016 Sharif University of Technology. All rights reserved.
\end{abstract}

\section{Introduction}

Three-Dimensional (3D) bio-imaging has many applications in medical diagnostics and therapy. Computerized Tomography (CT) is a powerful tool among existing bio-imaging techniques for capturing threedimensional bio-images. In fact, CT imaging systems have attracted great attention in last decades because of their fast and high-quality reconstruction, which results in low-complexity and low-cost hardware solutions.

In a CT scan procedure, several linear sensors receive X-ray radiations passed through the patient's body; then, special algorithms are utilized in order to reconstruct $2 \mathrm{D}$ and $3 \mathrm{D}$ images from the collected data. The quality of the reconstructed image is essentially influenced by the number of captured line projections. Nevertheless, gathering the required large amount of data requires the patient to be exposed to X-ray radiations for a long time. On the other side, the

\footnotetext{
*. Corresponding author. Tel.: +98 2166165927

E-mail address: Kavehvash@sharif.edu (Z. Kavehvash)
}

intensification of X-ray radiations potentially leads to ionization of body cells, which in turn raises the risk of cancer. Thus, one of the most important challenges in using the CT technique for biomedical imaging is to reduce the required samples without degrading the image quality.

Traditionally, image reconstruction requires a number of samples (measurements or observations), which are dictated purely by Nyquist limits. Due to the Nyquist constraint, image capturing with fewer samples than the Nyquist rate leads to a performance degradation. In traditional CT imaging systems, after data gathering in the form of radon coefficients, image reconstruction is performed through traditional algorithms such as Filtered Back Projection (FBP), which needs a complete set of radon coefficients determined by the Nyquist criteria. The main challenge of traditional algorithms is that by going under the Nyquist rate, the quality of the reconstructed image degrades significantly. For example, FBP $[1,2]$ is one of the renowned $\mathrm{CT}$ reconstruction algorithms requiring a large number of lownoise projections to yield an accurate reconstruction. Still, in a large number of 
applications, a complete projection cannot be obtained because of practical limitations such as short exposure time and detector's slip. To overcome these challenges, algebraic methods have been introduced, which suffer from high computational complexity, requiring a long time for reconstruction.

Recently, several works have been reported trying to reduce the sampling duration as well as image reconstruction time by interpolating the less-than-Nyquist captured samples [3]. These algorithms are based on the Fourier Slice Theorem (FST) to obtain data in the Fourier domain by mapping the 1D Fourier transform of radon measurements into the regular rectangular system. Yet, the estimation of Fourier samples on the rectangular system is errorprone due to subsequent interpolations. Furthermore, the density of radial points becomes sparser as one gets farther away from the center. This implies that the interpolation error of high-frequency components is greater than that of lowfrequency ones. To reduce the interpolation error, the sampling rate along the radial and angular directions should be chosen to be large enough. However, since a high sampling rate requires more projections, gathering enough measurements translates to additional exposure time and, thus, the main fundamental challenge still remains.

More recently, other solutions have been introduced, trying to reduce the CT scanning time with the aid of Compressive Sensing (CS). The compressive sensing concept utilizes the sparseness of the natural signals and images in different domains such as time, space, and frequency in order to reconstruct the desired signal with less-than-Nyquist samples [4-6]. Specifically, the Compressed Sensing (CS) method has found its application in $\mathrm{CT}$ imaging, being able to provide a low-radiation CT image reconstruction platform [7-13]. In fact, by using the CS approach in CT algorithms, the reconstruction process can be done with small number of data, reducing the health risk.

In a group of CS-based CT methods, Adaptive Steepest Descent Fourier Transform (ASD-FT) has been introduced that brings the projection data into the image's Fourier space by using FST, ensuring the consistency of the transformed projection data with Fourier transform of the reconstructed image [7]. However, this approach encounters some challenges in mapping data from polar coordinate into the regular rectangular system, which in turn degrades the quality of the reconstructed images. To solve this problem, ASD-Projection Onto Convex Sets (ASD-POCS) is proposed, which substitutes the Fourier transform with Gradient Magnitude Image (GMI) [8]. This approach employs the steepest-descent method, which suffers from a low convergence rate. Therefore, the slow convergence speed is the major drawback of the ASDPOCS algorithm.
Another problem of the steepest-descent method is dependence on the initialization points and parameters, meaning that if the optimality conditions are violated, it should be re-executed with new parameters.

Another group of CS-based CT image reconstruction approaches use Total Variation (TV) for image reconstruction with incomplete set of measurements [911]. Bian et al. exploited the sparsity of objects in the Total Variation (TV) domain, naming it the CSTV model [9]. This work was completed later in $[10,11]$ where the priori information was introduced as constraints that assisted the TV minimization formula to provide a high-quality image reconstruction.

Still, there are generally two difficulties in existing applications of CS to CT image reconstruction systems. The first problem arises from the fact that most CT images may not be piecewise smooth, thus not being sparse in the spatial domain. The second issue is that for a stable reconstruction, the fundamental CS theory requires the compressed sampling scheme to have an incoherence property in terms of the Restricted Isometry Property (RIP) of the CS matrix.

So far, there have been no results on the CS reconstruction of CT images under the RIP condition, though there are several works on the analysis of RIP [14] and empirical study of the incoherence property in terms of the phase transition map [15].

To solve these issues, recently, an adaptive CS approach has been introduced for the CT reconstruction, which updates the sampling matrix in each iteration [12]. The requirement for updating the sampling matrix in this approach obliges the patient to be under radiation of X-ray for a long time, which is contrary to the main goal of the exposure time reduction. In another recently reported work, a CT structure is proposed, which performs the compressive sampling in the Fourier domain [13]. This is done through random sampling in the Fourier domain, where the RIP constraint is better satisfied. Consequently, image reconstruction is done with a rate less than Nyquist while having a better image quality. However, the resulting image reconstruction performance is not adequate and the distortion of reconstructed image results in a non-smooth reconstruction output. This is mainly because of their random sampling in the radon Fourier domain, which has no guarantee for the consequent random sampling in spatial or Fourier domain of Cartesian coordinates. Also, this algorithm has high sensitivity to noise because of using ramp filter for weighting high frequency elements.

In this paper, a new CS-based CT image reconstruction is introduced in order to utilize the decent properties of the Fourier sampling in a more systematic manner. This is done by performing the compressive sampling based on the theory of partial Fourier sampling [16-18]. This sampling is possible by taking an 
advantage from the relation between radon coefficients and radial Fourier samples based on the Fourier slice theorem [19].

The paper is organized as follows. The proposed CT image reconstruction structure is addressed in Section 2, where also a brief review on the compressive radial Fourier sampling is presented. Section 3 presents simulation results, proving that the proposed approach reconstructs CT images with considerably low samples while, at the same time, keeping the image quality better than previous similar approaches. Finally, Section 4 concludes the paper.

\section{Proposed CT imaging structure}

As mentioned, the main foundation of this work is to minimize the CT scanning time through the partial Fourier sampling. Radial Fourier sampling is here possible because of the intrinsic relation between radial Fourier samples and the captured radon coefficients through the Fourier slice theorem [19]. Thus, in this section, first, the concept of the compressive sensing with specific emphasis on the radial Fourier sampling is briefly discussed and, then, the proposed structure will be introduced and analyzed.

\subsection{A short review on compressive sensing}

In the last decade, the concept of Compressive Sensing (CS) has opened new windows to reconstruct signals with fewer-than-Nyquist number of measurements through unifying sampling and compression process. In traditional compression techniques, a complete set of samples, dictated by the Nyquist rate, is acquired and then, the reconstructed image is compressed due to the limitations on the memory and transmission rate. In contrast, the compressive sensing approach tries to reduce the number of captured samples making the sampling faster and more efficient.

With respect to the sparsity of conventional signals in a specific domain, the reconstruction with a low data-rate is possible; thus, the sampling domain and sampling pattern are two of the main challenges for better CS reconstruction. In addition, another requirement for a successful compressive sampling approach is the satisfaction of the RIP condition. In other words, in order to guarantee the lossless image reconstruction through the CS theory, the acquisition matrix must satisfy the Restricted Isometry Property (RIP) $[5,20,21]$. The most well-known matrices satisfying the RIP condition with a high probability should have matrix elements drawn from Gaussian independent and identically distributed (iid) random numbers. Yet, another recently proposed high performance sampling pattern is the radial sampling in the Fourier domain [16-18].

A compressive sampling procedure can be mod- eled as follows:

$$
Y=\phi \times v
$$

where $y \in R_{(m \times 1)}$ is the incomplete set of samples to be captured, $\phi$ denotes the compressive sampling matrix that determines the method and structure of sampling such that $m<<n$, and $v \in R_{(n \times 1)}$ is the original signal that should be reconstructed.

The formulation in (1) is an ill-posed problem whose number of equations is less than the number of variables. Thus, without extra information, this problem does not converge. However if $v$ is a sparse signal, using this formula, it could be completely reconstructed with a high probability (see [5] for detail). Nevertheless, most natural images are not sparse in spatial domain while a majority of them have sparse representation in the Fourier or Wavelet domain. Therefore, the desired image, $u$, is sparse in a special basis like $v$, meaning that $v=\psi u$, where $v$ is the sparse representation of $u$ and $\psi$ is the transformation matrix, which transforms the image $u$ into the sparse basis $v$.

There exist various compressive sampling techniques, which yield a high-quality reconstructed image from a few number of captured samples. In fact, the sampling method determines the sampling pattern, $\phi$, and the sampling domain, $\psi$. Thus, Eq. (1) could be edited as follows:

$$
y=(\phi \psi) u \text {. }
$$

Incoherent property of $\phi$ and $\psi$ is one of the main constraints that should be satisfied for a proper signal reconstruction [5]. The reconstruction process is indeed an optimization procedure, which tries to minimize a well defined cost function based on Eq. (2) and the signal sparsity constraint.

It has been shown that an acceptable reconstruction can be obtained by using appropriate nonlinear recovery algorithms applied to only one part of the Fourier coefficients given on a set of $L$ radial lines in the Fourier plane [5]. In this case, the $\phi$ matrix has a pattern such as the one shown in Figure 1 where the $\psi$ matrix represents the Fourier transformation.

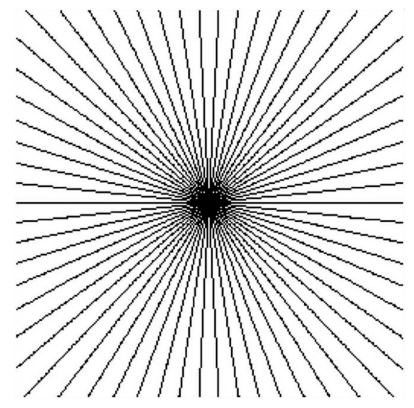

(a)

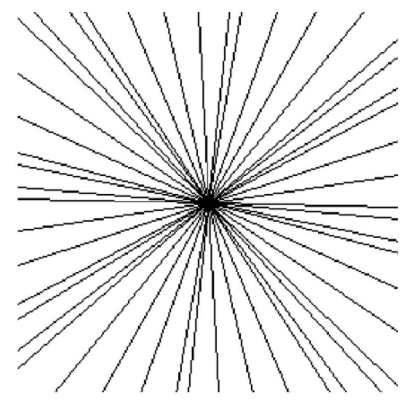

(b)
Figure 1. Two examples of radial sampling matrices. 
Apart from the satisfaction of RIP and IP constraints, in a Radial Fourier sampling scheme, lowfrequency components are sampled more densely than high-frequency components. This fact resembles the concept behind the common digital compression techniques where the most useful information from an object is gathered around the center of the Fourier plane.

Given that in a CT scan structure data are gathered as radon coefficients, the Radial Fourier sampling could be utilized due to the direct relations between radial Fourier samples and radon coefficients based on FST. This property is here utilized in order to perform CS-based CT imaging through the radial Fourier sampling where the RIP condition could be analyzed more systematically. The presented approach is described in the sequel.

\subsection{Proposed structure}

In this paper, the main goal is to implement a fast CSbased CT imaging structure based on radial Fourier sampling in order to satisfy the RIP condition more systematically. This is done through exploiting the relation between radon coefficients and radial Fourier samples. The main framework of the proposed structure for Radial Fourier CT scan is illustrated in Figure 2.

Let $I(x, y)$ represent a compactly supported continuous function on $R^{2}$ that shows an $m \times n$ image. The $\mathrm{CT}$ imaging system works based on capturing the radon samples. The radon transform, $\Re_{I}($.$) , is a function$ defined on the space of straight lines $L$ in $R^{2}$ by the line integral along each such line:

$$
\Re_{I}(L)=\int_{L} I(x)|d x| .
$$

Concretely, parametrization of any straight line, $L$, with respect to arc length, $t$, can always be written as:

$$
(x(t), y(t))=((t \sin \theta+s \cos \theta),(-t \cos \theta+s \sin \theta)),
$$

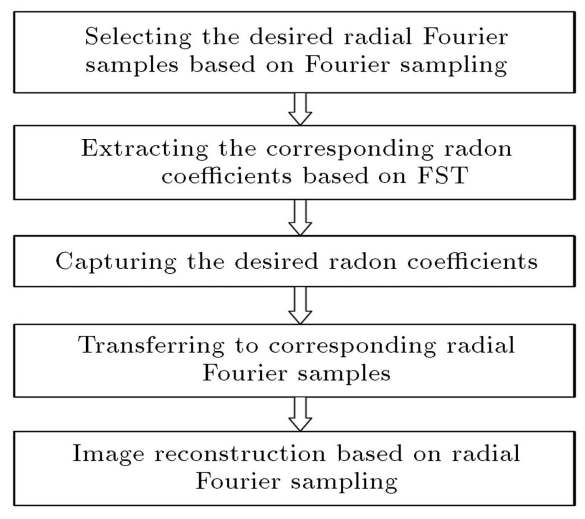

Figure 2. Proposed structure of the compressive sensing-based CT imaging structure. where $s$ is the distance of $L$ from the origin and $\theta$ is the angle between the normal vector to $L$ and the $x$ axis. It follows that the quantities $(s, \theta)$ can be considered as coordinates on the space of all lines in $R^{2}$, and the radon transform can be expressed in these coordinates by:

$$
\Re_{I}(s, \theta)=\iint I(x, y) \delta(x \cos \theta+y \sin \theta-s) d x d y
$$

Based on this, in order to make the CT scan procedure faster, we propose to reduce the number of samples through the partial Fourier sampling. To this end, we should first select the required radial Fourier samples of the object based on the chosen radial Fourier sampling pattern. This pattern has different models such as uniform or golden-angle as shown in Figure 1 [17]. The next step is to relate the selected radii in the Fourier domain to the corresponding radon coefficients through the Fourier slice theorem. With respect to FST, the 1D Fourier transform of line projections in an arbitrary angle, $\theta$, (named as $F_{R}(\omega, \theta)$ ) is equal to a $1 \mathrm{D}$ slice of the 2D Fourier transform of the image in the same angular direction. This theorem could be modeled as follows:

$$
F_{\Re}(\omega, \theta)=F_{I}(\omega \cos \theta, \omega \sin \theta),
$$

where $F_{I}($.$) represents the 2 \mathrm{D}$ Fourier transform of the original image. With this relation in hand, the corresponding required radon coefficients, $\Re_{I}(s, \theta)$, could be obtained from the selected radial Fourier samples. Then, the CT structure scans the object to capture the obtained desired radon coefficients.

The schematic of the recording procedure is shown in Figure 3. These captured radon coefficients should be converted to the corresponding radial Fourier samples in order to run the CS optimization step and reconstruct the full image. However, before performing the conversion procedure, the radon coefficients should undergo a zero-padding step as shown in Figure 3, where the schematic of the capturing process for obtaining the required Fourier radii is demonstrated. The 1D Fourier transforms of the zero-padded radon coefficients are computed in the next step, which yield the required Fourier radii.

With these radial Fourier samples in hand, the optimization procedure based on the wellknown method of alternating direction of multiplier [22] is run in order to reconstruct the whole image in the Cartesian coordinate. After choosing the optimization method, the final goal is to find the image, which has the minimum total variation with the maximum accuracy. This goal results in the following minimization problem:

$$
\min \Sigma_{i}\left\|D_{i} u\right\|_{2}+\tau|\psi u|+\mu / 2\|\phi \psi u-y\|_{2}^{2}
$$




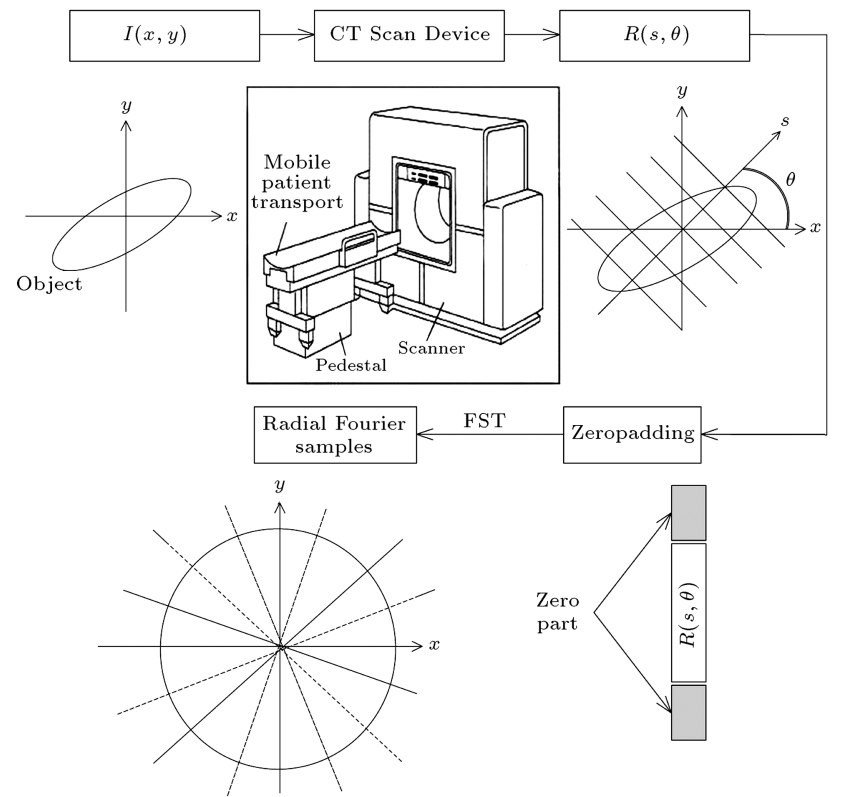

Figure 3. The obtained radial Fourier samples from the radon coefficients.

where the first term is the discretization of the Total Variation (TV) of $u$ over its all pixels across vertical and horizontal directions, the second term is 11-norm of the sparse representation of $u$ under $\psi$, and the last term denotes the relaxation term. Moreover, $\tau$ and $\mu$ are regularization parameters. The main challenge to find the solution of Relation (7) is the non-differentiability of its first and second terms. By defining two auxiliary variables $z$ and $w$, Relation (7) can be rewritten as:

$$
\begin{aligned}
& \min \Sigma_{i}\left\|w_{i}\right\|_{2}+\tau|\psi z|+\mu / 2\|\phi \psi u-y\|_{2}^{2}, \\
& \text { s.t. } w_{i}=D_{i} u \quad \forall \quad i, z=\psi^{T} u .
\end{aligned}
$$

In order to solve Relation (8) linearly, the augmented Lagrangian term is used as the minimization goal. By means of the augmented Lagrangian of Relation (8) and using an Alternating Direction Method (ADM), this equation could be simply converted to a least squares problem. Therefore, the reconstruction time is decreased by using ADM algorithm for solving the optimization problem. The algorithm uses one- and two-dimensional shrinkage to compute $w$ and $z$ in each iteration in parallel. Then, the value of $u$ is updated until the relative error becomes small enough (less than a predefined threshold value) (see [22] for details).

Therefore, there are three main tasks in each iteration for the ADMM algorithm: computing the variables $z$ and $w$, obtaining a new output, and updating the required variables for the next iteration. To support our proposed CT imaging structure, we design a VLSI architecture for the reconstruction algorithm, which provides a real-time prototype. The block diagram of its top module is depicted in Figure 4(a). It is composed of three processing units and one memory management unit, working in accordance with a control unit. The control unit sends the necessary control signals to the processing units to initiate their operations, and generates the address values to do the read and write operations on the memory unit. In addition, this design includes the infrastructure overheads such as memory and Ethernet controller to run on the Xilinx Virtex-6 FPGA ML605 Evaluation Kit. The overall data-path of this design is shown in Figure 4(b).

Thus, the proposed structure reduces the sampling points significantly compared to common scanning structures such as FBP, which in turn reduces the whole scanning time.

In order to quantitatively demonstrate the improved performance of the proposed structure, three metrics are utilized in this paper. The first metric is the Mean Square Error (MSE) between the original, $I_{O}$, and reconstructed, $I_{R}$, images with the following formula:

$$
\mathrm{MSE}=E\left[\left(I_{R}-I_{O}\right)^{2}\right] .
$$

The second evaluation metric is the Peak Signal-to-

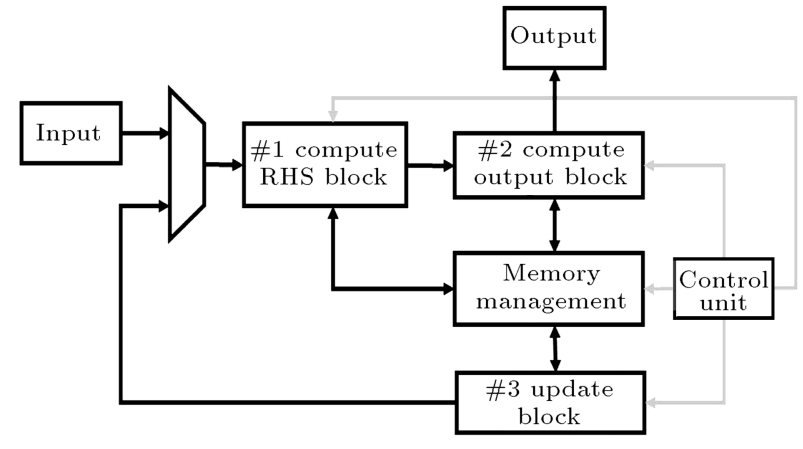

(a)

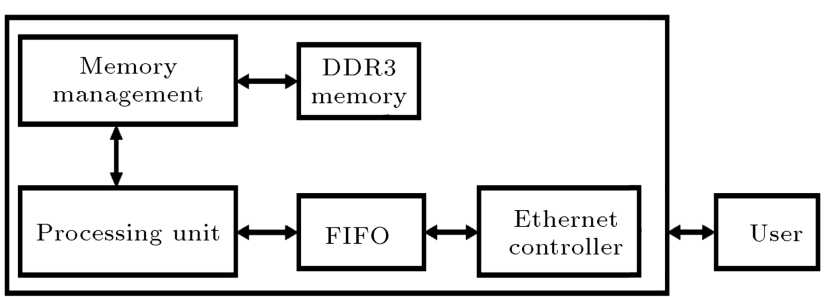

(b)

Figure 4. Hardware design: (a) The block diagram of the hardware design; (b) the overall data-path of the hardware design. 
Noise Ratio (PSNR) of the reconstructed image. The PSNR metric is defined as follows:

$$
\operatorname{PSNR}=20 \log \left(\frac{I}{\sqrt{(\mathrm{MSE})}}\right)
$$

where $I$ represents the maximum intensity of the reference image. PSNR results are complemented by another well-known evaluation metric, i.e. Structure Similarity Index Metric (SSIM), which measures the similarity between the original and reconstructed images [23]. The SSIM metric is defined as follows:

$$
\operatorname{SSIM}=\frac{\left(2 m_{I_{1}} m_{I_{2}}+c_{1}\right)\left(2 \sigma_{I_{1} I_{2}}+c_{2}\right)}{\left(m_{I_{1}}^{2}+m_{I_{2}}^{2}+c_{1}\right)\left(\sigma_{I_{1}}^{2}+\sigma_{I_{2}}^{2}+c_{2}\right)},
$$

where $m_{I}$ and $\sigma_{I}$ represent the image mean and variance, respectively, $\sigma_{I_{1} I_{2}}$ shows the covariance of the original and reconstructed images, and $c_{1}$ and $c_{2}$ are stabilization parameters.

In order to demonstrate superiority of the proposed structure in reducing the $\mathrm{CT}$ scan time while, at the same time, yielding a high quality image, exhaustive simulations are performed, reported in the next section. The performed simulations compare the quality of the proposed method with those of other interpolation-based and CS-based approaches, intended to reduce the $\mathrm{CT}$ scan time.

\section{Results and compariosn}

In this section, the proposed structure is compared with three other CT image reconstruction methods, which try to lessen the CT scan time. These methods are CSTV [9], interpolation [3], and WL1 [13]. The CT image of a sample SheppLogan phantom is obtained through MATLAB simulations. The considered sample is an image of size $256 \times 256$ pixels while the value of the regularization parameters in each algorithm is selected empirically. The reconstructed image resulted from each algorithm is shown in Figure 5. As it is clear from the colormap results of MATLAB and enlarged part of each image, shown in Figure 6, for CS-based algorithms, the proposed structure performs better in saving the image high-frequency contents while, at the same time, not adding considerable distortion.

In order to evaluate the reconstructed images based on these metrics, two simulation scenarios were performed. In the first simulation, the reconstruction process was done with 5 different algorithms including CSTV, WL1, FBP, interpolation, and the proposed algorithm, where the quality of the reconstructed images versus the number of projections was fully compared. The evaluated PSNR for this simulation is shown in Figure 7 . This figure clearly demonstrates the superior performance of the proposed method based on PSNR metric.

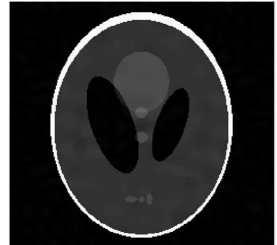

(a)

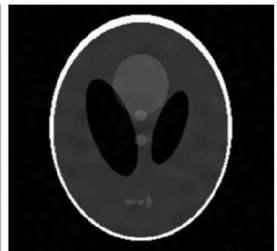

(b)

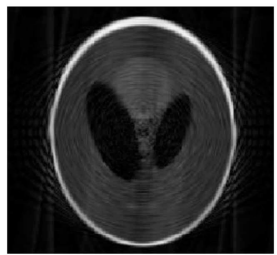

(d)

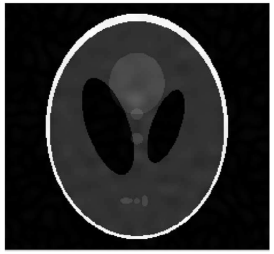

(c)
Figure 5. Reconstructed images with 24 projections: (a) Proposed structure with uniform angelprojection (PSNR $=42.42)$; (b) CSTV result $(\mathrm{PSNR}=40.47)$; (c) WL1 result (PSNR $=40.83)$; and $(\mathrm{d})$ interpolation result $(\mathrm{PSNR}=19.47)$.

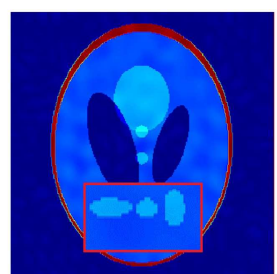

(a)

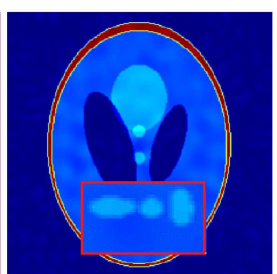

(b)

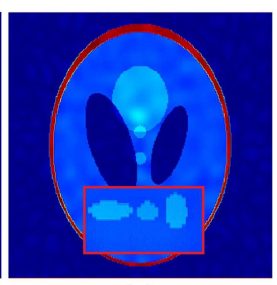

(c)
Figure 6. Reconstructed images with JET colormap: (a) Proposed structure with uniform angel projection; (b) CSTV result with distorted high frequency contents; and (c) WL1 result with added distortions apparent in upper circle.

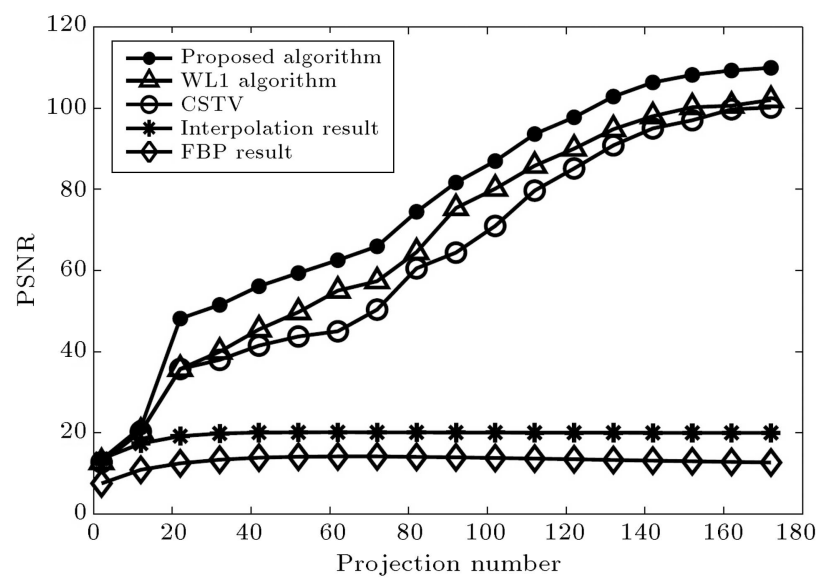

Figure 7. PSNR of different algorithms with changing projection numbers.

In the second simulation scenario, the results of the CS-based CT image reconstruction algorithms including CSTV, WL1, and our proposed method are compared for various numbers of iterations. The measured PSNR values for all algorithms are depicted versus the iteration numbers in Figure 8 . It is clear 


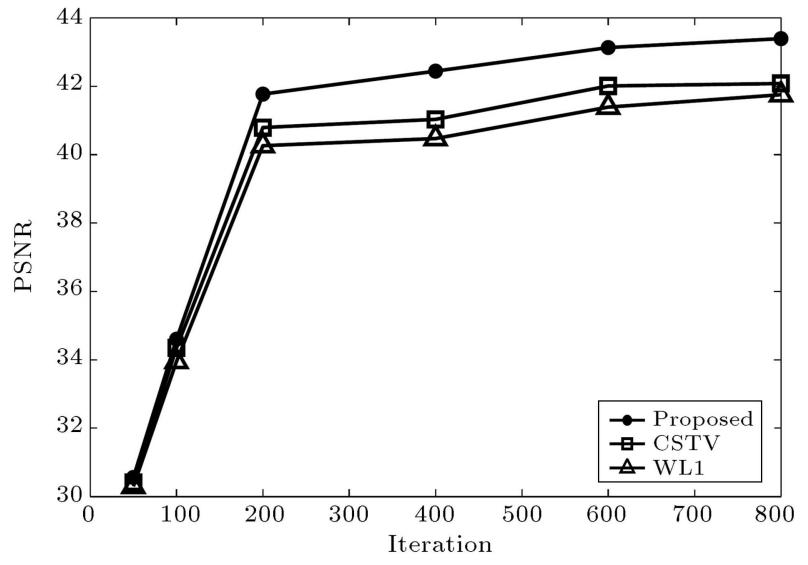

Figure 8. PSNR of different algorithms with changing iteration numbers.

that the proposed structure has better quality with the same number of iterations, i.e. in 400 iterations.

With regard to this figure, it is obvious that the proposed method performs better based on the PSNR metric for different iteration numbers. Furthermore, the superior performance of the image reconstructed from the proposed structure is also confirmed through SSIM and MSE metrics. The numerical results are shown in Table 1. As it is clear in this table, the result of the proposed structure is better than the other result (about $4 \%$ in PSNR). Also, this structure has the minimum MSE and better SSIM.

As mentioned in the Introduction, the high sensitivity to noise is one of the important shortcomings of WL1. In contrast, CSTV and the proposed structure have low sensitivity to noise by using the total variation and minimizing the gradient of image. Figure 9 shows the results of reconstructed images with noisy data. As can be seen clearly, distortion in the texture of the result of WL1 is obvious while CSTV and the proposed structure perform better in the presence of noise.

Finally, in order to have a more reliable comparison between the performances of the proposed structure and other CS-based methods, real projection data are employed and analyzed. Figure 10 shows the reconstructed images with real dataset [24]. As is clear from this figure, the proposed algorithm has a better reconstruction quality than CSTV and WL1 algorithms. CSTV has difficulty in the reconstruction of edges due to the nature of the total variation and WL1 has distortion in the specified parts.

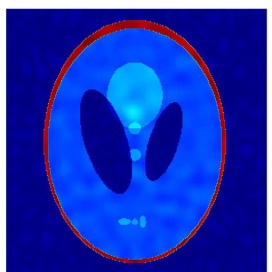

(a)

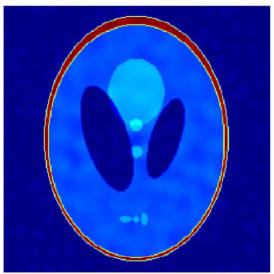

(b)

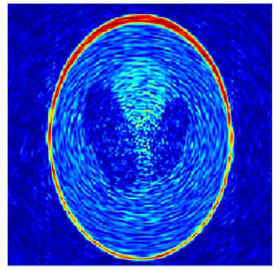

(d)

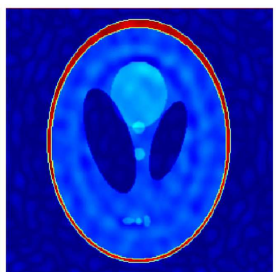

(c)
Figure 9. Reconstructed images with noisy data: (a) Proposed structure with uniform angel projection (PSNR $=36.45)$; (b) CSTV result $(\mathrm{PSNR}=38.38)$; (c) WL1 result $(\mathrm{PSNR}=31.11)$; and $(\mathrm{d})$ DFT result $(\mathrm{PSNR}=$ 18.39).

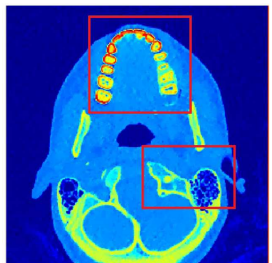

(a)

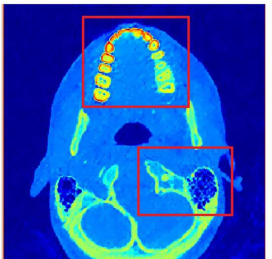

(b)

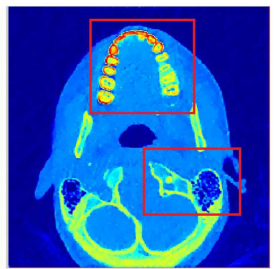

(c)
Figure 10. Reconstruction results with real dataset (250 iteration): (a) Proposed structure with uniform angel projection $(\mathrm{PSNR}=32.95)$; (b) CSTV result $(\mathrm{PSNR}=$ $29.45)$; and (c) WL1 result $(\mathrm{PSNR}=31.08)$.

In summary, both the qualitative visual performance and the quantitative metrics confirm the superiority of the proposed CS-based CT imaging structure in preserving the image quality while, at the same time, lessening the CT scan time.

\section{Conclusion}

In this paper, an efficient CT imaging structure based on compressive sensing is proposed in order to mitigate the CT scanning time. In order to satisfy the requisite of the compressive sampling algorithms in a systematic manner, partial Fourier sampling was utilized. Thanks to the intrinsic relation between captured radon samples and radial Fourier samples,

Table 1. Comparison of the results of different CS-based algorithms (24 line projection and 400 iteration).

\begin{tabular}{cccccc}
\hline & This Work & CSTV [9] & WL1 [13] & FBP & Interpolation \\
\hline PSNR & 42.42 & 40.47 & 40.83 & 13.43 & 17.81 \\
MSE & $5.6989 e^{-05}$ & $8.9741 e^{-05}$ & $7.9831 e^{-05}$ & 0.0315 & 0.0746 \\
SSIM & 0.9999 & 0.9998 & 0.9999 & -1 & 0.9992 \\
\hline
\end{tabular}


Partial (radial) Fourier sampling could be implemented more systematically. This systematic implementation helped in better satisfaction of CS conditions, such as Restricted Isometry Property (RIP). Simulations were performed, in which the performance of the proposed structure was compered with those of 3 other approaches that also tried to lessen CT scan time through either CS or interpolations. The presented qualitative and quantitative results showed the superior performance of the proposed structure.

\section{Acknowledgment}

We would like to thank Amir Ashkan Farsaei for his very helpful comments on a draft of hardware design parts of this article.

\section{References}

1. Bracewell, R.H. and Riddle, A.C. "Inversion of fan beam scans in radio astronomy", Astrophysics Journal, 150, pp. 427-434 (1967).

2. Ramanchandran, G.N. and Lakshminarayanan, A.V. "Three dimensional reconstructions from radiographs and electron micrographs: Application of convolution instead of Fourier transforms", Proceedings of the $\mathrm{Na}$ tional Academy of Sciences, 68, pp. 2236-2240 (1971).

3. Stark, H., Woods, J. and Paul, I., "Direct Fourier reconstruction in computer tomography", IEEE Trans., Speech and Signal Processing, 29, pp. 237-245 (Apr. 1981).

4. Donoho, D.L. "Compressed sensing", IEEE Trans. Inform. Theory, 52, pp. 1289-1306 (2006).

5. Candes, E., Romberg, J. and Tao, T. "Robust uncertainty principles: exact signal reconstruction from highly incomplete frequency information", Information Theory, IEEE Transactions on, 52, pp. 489-509 (Feb. 2006).

6. Candes, E. and Tao, T. "Near-optimal signal recovery from random projections: Universal encoding strategies?", Information Theory, IEEE Transactions on, 52, pp. 5406-5425 (Dec. 2006).

7. LaRoque, S.J., Sidky, E.Y. and Pan, X. "Accurate image reconstruction from few-viewand limited-angle data in diffraction tomography", Journal of the Optical Society ofAmerica, 25(7), pp. 1772-1782 (Jun 2008).

8. Sidky, E.Y. and Pan, X.C. "Image reconstruction in circular cone-beam computed tomography by constrained, total-variation minimization", Physics in Medicine and Biology, 53, pp. 777-807 (2008).

9. Bian, J., Wang, J., Han, X., Sidky, E.Y., Shao, L. and Pan, X. "Optimization-based image reconstruction from sparse-view data in offset-detector CBCT", Physics in Medicine and Biology, 58, pp. 205-230 (2013).
10. Lauzier, P.T. and Chen, G.H. "Characterization of statistical prior image constrained compressed sensing. I. Applications to time-resolved contrast-enhanced CT", Medical Physics, 39, pp. 5930-5948 (2012).

11. Lauzier, PT. and Chen, GH. "Characterization of statistical prior image constrained compressed sensing (PICCS): II. application to dose reduction", Medical Physics, 40 (2013).

12. Barkan, O., Weill, J., Averbuch, A. and Dekel, S. "Adaptive compressed tomography sensing", IEEE Conferance, Computer Vision and Pattern Recognition (CVPR), pp. 2195-2202 (2013).

13. Hou, W. and Zhang, C. "A Compressed sensing approach to low-radiation CT reconstruction", Communication Systems, Networks \& Digital Signal Processing (CSNDSP), pp. 793-797 (2014).

14. Jrgensen, J.H., Sidky, E.Y. and Pan, X. "Quantifying admissible undersampling for sparsity-exploiting iterative image reconstruction in X-ray CT", IEEE Transactions on Medical Imaging, 32, pp. 460-473 (2013).

15. Jrgensen, J.H., Sidky, E.Y., Hansen, P.C. and Pan, X. "Quantitative study of undersampled recoverability for sparse images in computed tomography", Arxiv, pp. 120 (2012).

16. Stern, A. "Compressed imaging system with linear sensors", Opt. Lett., 32, pp. 3077-3079 (Nov. 2007).

17. Evladov, S., Levi, O. and Stern, A. "Progressive compressive imaging from radon projections", Opt. Xpress, 20, pp. 4260-4271 (Feb. 2012).

18. Yang, J., Zhang, Y. and Yin, W. "A fast alternating direction method for tvl1-12 signal reconstruction from partial fourier data", Selected Topics in Signal Processing, IEEE Journal of, 4, pp. 288-297 (April 2010).

19. Hsieh, J., Computed Tomography: Principles, Design, Artifacts, and Recent Advances, Bellingham, WA: SPIE (2009).

20. Duarte, M., Davenport, M., Takhar, D., Laska, J., Sun, T., Kelly, K. and Baraniuk, R. "Single-pixel imaging via compressive sampling”, Signal Processng Magazine, IEEE, 25, pp. 83-91 (March 2008).

21. Willett, R.M., Marcia, R.F. and Nichols, J.M. "Compressed sensing for practical optical imaging systems: a tutorial", Optical Engineering, 50, no. 7, pp. 072601072601-13 (2011).

22. Yang, J., Zhang, Y. and Yin, W. "A fast alternating direction method for tvl1-12 signal reconstruction from partial fourier data", Selected Topics in Signal Processing, IEEE Journal of, 4, pp. 288-297 (April 2010).

23. Wang, Z., Bovik, A.C., Sheikh, H.R. and Simoncelli, E.P. "Image quality assessment: From error visibility to structural similarity", Trans. Img. Proc., 13, pp. 600-612 (Apr. 2004). 
24. DICOM sample image sets (http://www.osirixviewer.com/datasets/).

\section{Biographies}

Hasan Abbasi received his BS degree in Electrical Engineering in 2013 from Shahid Beheshti University, Tehran, Iran, and MSc degree in 2015 from Sharif University of Technology. He is currently PhD student at Sharif University of Technology. His areas of interest and experience are medical imaging and signal processing.

Zahra Kavehvash received her BS degree in 2005 and PhD degree in 2013, both in Electrical Engineering from Sharif University of Technology, Iran. She is interested in 3D imaging, millimeter-wave imaging and holography, and biomedical imaging systems. Dr. Kaveh vash joined the Department of Electrical Engineering at Sharif University of Technology, Tehran, as an Assistant Professor in 2013.

Mahdi Shabany received his BS degree in Electrical Engineering from Sharif University of Technology, Iran, in 2002. He finished his MS and PhD degrees in Electrical Engineering at Toronto University in 2009. He is interested in VLSI implementation of biomedical signal processing algorithms, architecture/algorithm design for $5 \mathrm{G}$ communication systems, and ASIC/RTL digital circuit design. Dr. Shabany joined the Department of Electrical Engineering, Sharif University of Technology, Tehran, as an Assistant Professor in 2010. 